\title{
Near infrared photoimmunotherapy prevents lung cancer metastases in a murine model
}

\author{
Kazuhide Sato ${ }^{1}$, Tadanobu Nagaya ${ }^{1}$, Yuko Nakamura ${ }^{1}$, Toshiko Harada ${ }^{1}$, \\ Peter L. Choyke ${ }^{1}$, Hisataka Kobayashi ${ }^{1}$ \\ ${ }^{1}$ Molecular Imaging Program, Center for Cancer Research, National Cancer Institute, Bethesda, MD 20892-1088, USA.
}

Correspondence to:

Hisataka Kobayashi, e-mail: Kobayash@mail.nih.gov

Keywords: near infrared photoimmunotherapy, metastasis prevention, targeted metastasis treatment, HER2 receptor

Received: February 25, $2015 \quad$ Accepted: April 30, $2015 \quad$ Published: May 13, 2015

\section{ABSTRACT}

Near infrared photoimmunotherapy (NIR-PIT) is a new cancer treatment that combines the specificity of intravenously injected antibodies with the acute toxicity induced by photosensitizers after exposure to NIR-light. Herein, we evaluate the efficacy of NIR-PIT in preventing lung metastases in a mouse model. Lung is one of the most common sites for developing metastases, but it also has the deepest tissue light penetration. Thus, lung is the ideal site for treating early metastases by using a light-based strategy. In vitro NIR-PIT cytotoxicity was assessed with dead cell staining, luciferase activity, and a decrease in cytoplasmic GFP fluorescence in 3T3/ HER2-luc-GFP cells incubated with an anti-HER2 antibody photosensitizer conjugate. Cell-specific killing was demonstrated in mixed 2D/3D cell cultures of 3T3/HER2-lucGFP (target) and 3T3-RFP (non-target) cells. In vivo NIR-PIT was performed in the left lung in a mouse model of lung metastases, and the number of metastasis nodules, tumor fluorescence, and luciferase activity were all evaluated. All three evaluations demonstrated that the NIR-PIT-treated lung had significant reductions in metastatic disease ( $* p<0.0001$, Mann-Whitney $U$-test) and that NIR-PIT did not damage nontarget tumors or normal lung tissue. Thus, NIR-PIT can specifically prevent early metastases and is a promising anti-metastatic therapy.

\section{INTRODUCTION}

Cancer metastasis is the main cause of cancer death. After patients develop metastatic disease in lung, the treatment options become limited to palliative surgical resection of visible oligometastases or systemic chemotherapy with more advanced disease [1, 2]. Once established, metastatic foci are even more difficult to treat because they acquire their own blood supply and microenvironment [2]. Thus, a therapy that could treat early lung metastases with minimal damage to the surrounding normal organs would be welcome.

The concept of targeted light therapy with photosensitizers was established over three decades ago $[3,4]$. However, most photosensitizers do not target tumors with sufficient selectivity resulting in off-target side effects. Attempts to improve photosensitizers have been limited by the hydrophobicity of traditional photosensitizers used in photodynamic therapy (PDT), which dominate the pharmacokinetics of antibody-PDT conjugates. However, if the photosensitizer is highly hydrophilic, then the pharmacokinetics of an antibodyphotosensitizer conjugate are governed by the antibody, and true targeting is possible. IRDye700DX (IR700, a silica-phthalocyanine dye) is a highly hydrophilic photosensitizer that is excited by near infrared (NIR) light at a wavelength of $690 \mathrm{~nm}$. If it is conjugated to a monoclonal antibody, this antibody photosensitizer conjugate (APC) is able to treat tumors, a process that has been called "near infrared photoimmunotherapy" (NIRPIT). This treatment differs from traditional PDT not only in the hydrophilicity of the photosensitizer but also in its reliance on NIR-light that has better tissue penetration than lower wavelength light used with PDT. NIR-PIT APCs demonstrate similar intravenous pharmacokinetics to naked antibodies, which results in highly targeted accumulation with minimal non-target binding. If they are bound to targeted cells and exposed to $690 \mathrm{~nm}$ NIR-light, these APCs induce rapid, selective and irreversible damage to the cell membrane. In vitro studies have demonstrated 
that NIR-PIT is highly target cell-specific; therefore, non-target expressing cells suffer no toxic effects, even if they are immediately adjacent [5]. Cell membrane rupture can be demonstrated within minutes of exposure to NIR-light in targeted cells $[6,7]$. Because of its high selectivity and minimal side effects as well as the excellent transmission of NIR-light in lung tissue, NIR-PIT is a potential therapy to prevent further growth of early lung metastases. Here, we investigate the efficacy of NIR-PIT in a murine model of early-stage lung metastases.

\section{RESULTS}

\section{Characterization of cell line and target-specific cell killing with NIR-PIT in in vitro $2 \mathrm{D}$ culture}

To monitor the effect of NIR-PIT, the Balb/3T3 cell line was modified to express HER2, GFP and luciferase (3T3/HER2-luc-GFP). As a non-target of NIR-PIT, the Balb/3T3 cell line was optically modified to express RFP (3T3-RFP) (Figure 1A). The antibody-photosensitizer conjugate, trastuzumab-IR700 (tra-IR700), was incubated with 3T3/HER2-luc-GFP or 3T3-RFP cells, and fluorescence signals were evaluated by flow cytometry. After a 6-hr tra-IR700 incubation, 3T3/HER2-luc-GFP cells showed high fluorescence and were blocked by excess trastuzumab, which suggested specific binding. Almost no binding of tra-IR700 was observed in 3T3-RFP cells (Figure 1B). Serial fluorescence microscopy of 3T3/HER2-luc-GFP cells was performed before and after NIR-PIT with $690 \mathrm{~nm}$ light. After exposure to $2 \mathrm{~J} / \mathrm{cm}^{2}$ NIR-light, cellular swelling and bleb formation were observed (Figure 1C). Time-lapse image analysis showed acute morphologic changes in the cell membrane, and cells incubated with propidium iodide (PI) demonstrated fluorescence of PI, which indicated cell death (Supplemental videos 1,2). Most of these cellular changes were observed within 25 min of light exposure, which indicated the rapid induction of necrotic cell death after NIR-PIT. To quantify the effect of in vitro NIR-PIT, a cytotoxicity assay consisting of PI staining and luciferase activity was performed $1 \mathrm{hr}$ after NIR-light irradiation. Based on the incorporation of PI, the cell death percentage increased in a light dose-dependent manner. No significant cytotoxicity was observed with either NIR light exposure or APC alone (Figure 1D). Bioluminescence showed significant decreases in relative light units (RLU) in NIRPIT treated cells but not in controls (Figure 1E, 1F). BLI also showed a decrease in luciferase activity in a light dose-dependent manner (Figure 1F). GFP fluorescence intensity was greatly reduced in dead cells (stained positive with PI), whereas GFP fluorescence was preserved in surviving cells (Figure 1G). GFP fluorescence was reduced after NIR-PIT because the GFP was extruded from the cytoplasm after membrane rupture and was therefore markedly diluted and/or denatured. The GFP diminished cells increased in a light dose-dependent manner, and no significant change was detected with NIR-light exposure or APC alone based on FACS analysis (Figure 1H). Taken together, these data suggest that the effects of NIR-PIT on 3T3/HER2-luc-GFP could be monitored with both GFP fluorescence and bioluminescence in vivo. Next, to demonstrate selective elimination of target-expressing cells, a 2D mixed cell culture consisting of 3T3/HER2luc-GFP and 3T3-RFP was used. Selective cell killing of 3T3/HER2-luc-GFP was documented with Cytox dead cell staining, and no significant changes were observed in HER2-negative 3T3-RFP cells after exposure to NIRlight, which suggested that NIR-PIT induced no damage in non-target cells that were immediately adjacent to target cells (Figure 1I). Elimination of 3T3/HER2-luc-GFP cells from an almost-confluent 2D mixed cell culture was demonstrated after NIR-PIT (Figure 1J).

\section{Characterization of in vitro 3D culture and target specific cell killing with NIR-PIT in a mixed 3D culture}

Next, we evaluated the efficacy of NIR-PIT in 3D spheroids (Figure 2). Fluorescence images of 3T3/HER2luc-GFP or 3T3-RFP spheroids at day 3 or day 7 were obtained (Figure 2A). NIR-PIT caused necrotic cell death in the APC-bound outer layer of the spheroid (Figure 2B). After exposure to NIR-light, the spheroid immediately expanded, and the outer cells became necrotic, as demonstrated by staining with PI (Supplemental videos 3 , 4). These changes occurred within $25 \mathrm{~min}$ of light exposure. To demonstrate target specific cell killing in the $3 \mathrm{D}$ cell culture, a mixed 3D spheroid that consisted of the two described cell lines was established (Figure 2C). Daily repeated NIR-PIT (Figure 2D) achieved complete eradication of the 3T3/HER2-luc-GFP cell population (diminishing GFP fluorescence) in the mixed 3D spheroid, and 3T3-RFP cells and other controls continued to grow (Figure 2E), as confirmed by BLI $(* p=0.0092<0.01$, $* * p<0.0001$ vs control, Tukey's test with ANOVA) (Figure 2F, 2G). These results suggest that repeated NIRPIT could eradicate target-expressing cells (3T3/HER2luc-GFP) that were growing in mixed 3D spheroids without damaging non-target cells (3T3-RFP).Unlike 2D cultures in which the APC could infiltrate the entire cell mixture and in which one NIR exposure was sufficient, 3D cultures required repeated NIR exposure because the APC entered new parts of the spheroid after every treatment.

\section{NIR-PIT prevents formation of metastatic disease in vivo}

In vivo mouse models of lung metastases consisting of 3T3-RFP, 3T3/HER2-luc-GFP, and mixtures of 3T3RFP and 3T3/HER2-luc-GFP were established and monitored by fluorescence imaging and BLI (Figure 3A). 
A

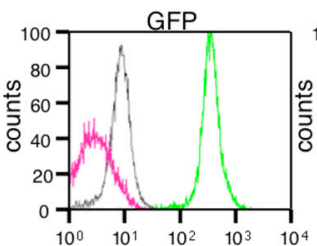

$\mathrm{C}$

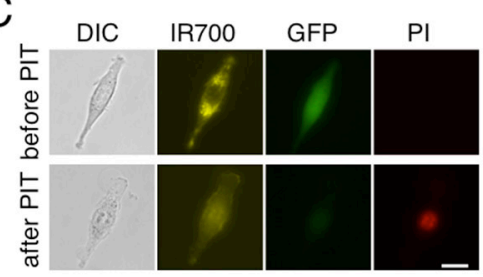

$\mathrm{F}$

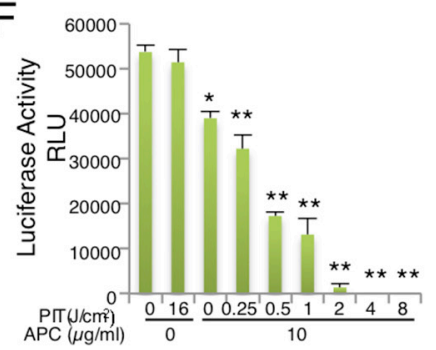

$\mathrm{H}$

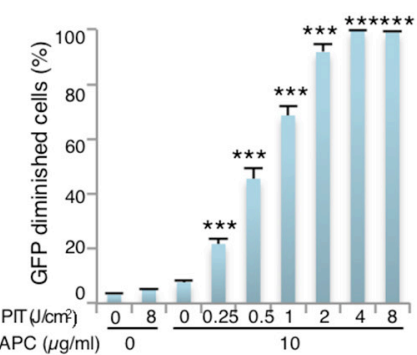
$\operatorname{APC}(\mu g / m) \frac{0}{0} 0$

$\mathrm{G}$

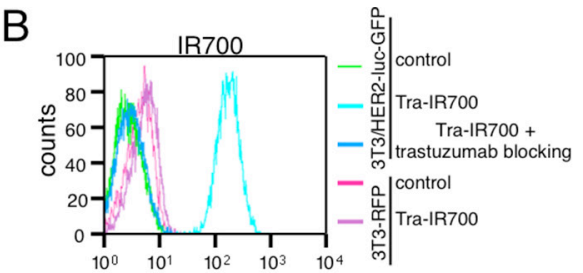

D
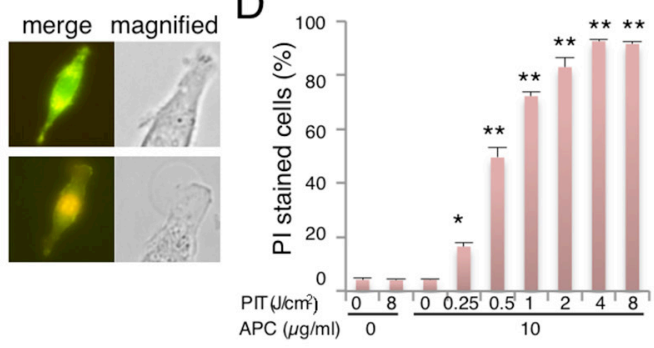

E
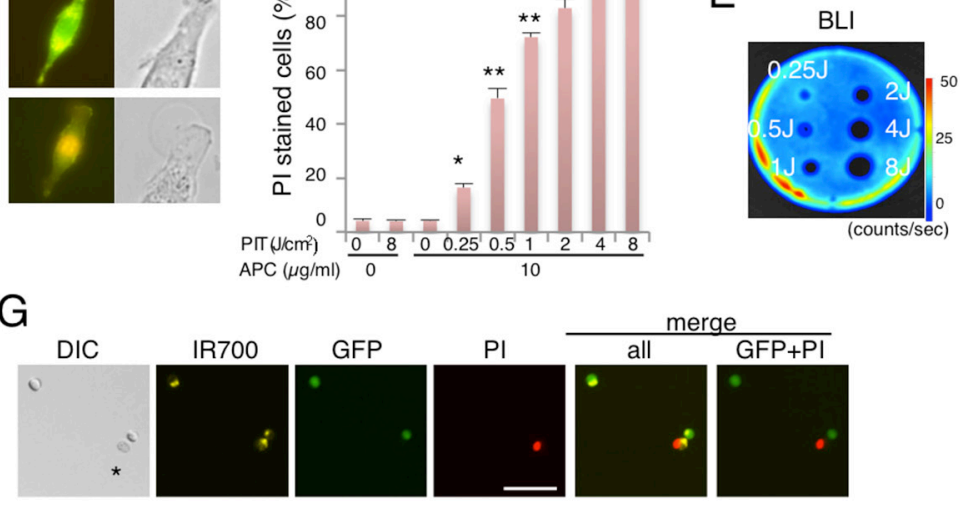

$J$
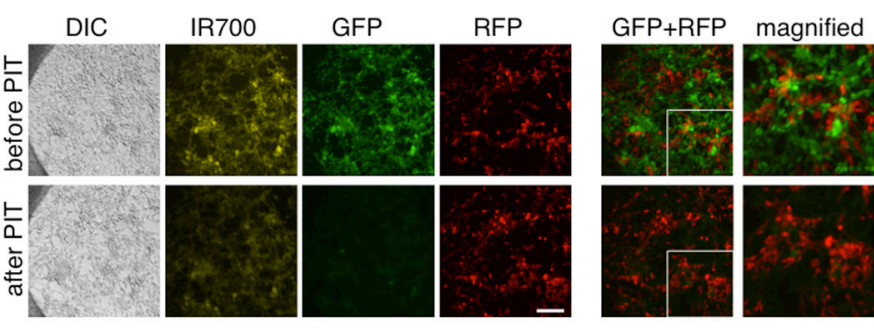

I
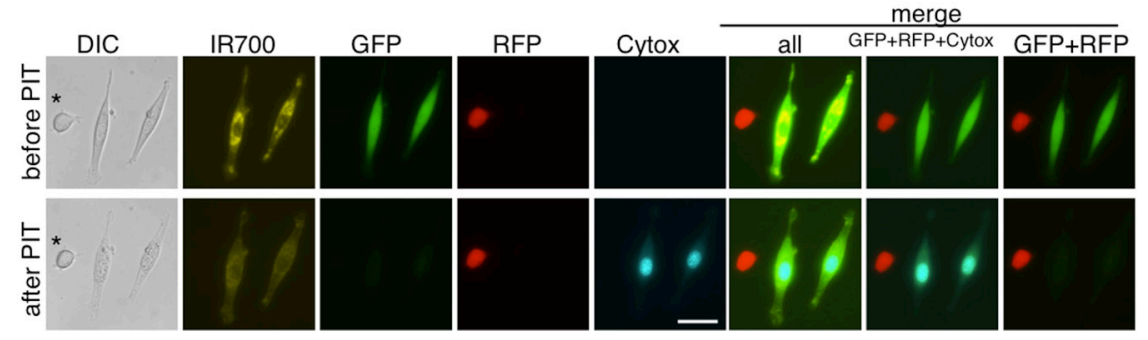

Figure 1: Effect of NIR-PIT on 2D cell monoculture and mixed cell culture. A. Characterization of 3T3/HER2-luc-GFP cells and 3T3-RFP cells. B. 3T3/HER2-luc-GFP cells demonstrate HER2 expression. Specific binding was demonstrated in a blocking study with excess trastuzumab. Non-HER2 expressing Balb/3T3-RFP cells were also examined with tra-IR700 incubation, which confirmed no binding. C. 3T3/HER2-luc-GFP cells were incubated with tra-IR700 for $6 \mathrm{hr}$ and observed before and after irradiation with NIR-light $\left(2 \mathrm{~J} / \mathrm{cm}^{2}\right)$. Necrotic cell death was observed after exposure to NIR-light (1 hr after NIR-PIT). PI staining indicates necrotic cell death. Bar $=50 \mu \mathrm{m}$. D. Membrane damage and necrosis induced by NIR-PIT were measured by dead cell count using PI staining. Cell killing increased in a NIR-light dose-dependent manner $\left(n=4,{ }^{*} p<0.001,{ }^{*} p<0.0001\right.$, vs. untreated control, Student's $t$ test). E. Bioluminescence imaging (BLI) of a treated $10 \mathrm{~cm}$ cell culture dish demonstrated that luciferase activity in 3T3/HER2-luc-GFP cells decreased in a NIRlight dose-dependent manner. F. Luciferase activity in 3T3/HER2-luc-GFP cells was measured as relative light unit (RLU), which also decreased in a NIR-light dose-dependent manner $\left(n=4,{ }^{*} p<0.001,{ }^{* *} p<0.0001\right.$, vs. untreated control, Student's $t$ test). G. 3T3/HER2luc-GFP cells were incubated with tra-IR700 for $6 \mathrm{hr}$ and irradiated with NIR-light $\left(0.25 \mathrm{~J} / \mathrm{cm}^{2}\right)$. GFP-fluorescence intensity decreased in dead cells (*) stained by PI but was unchanged in living cells at $1 \mathrm{hr}$ after NIR-PIT. Bar $=200 \mu \mathrm{m}$. H. GFP fluorescence intensity decreased after NIR-PIT in a NIR-light dose-dependent manner $\left(n=4,{ }^{* * *} p<0.0001\right.$, vs. untreated control, Student's $t$ test). I. 3T3/HER2-luc-GFP cells were co-cultured with 3T3-RFP (non-HER2 expressing) cells. The mixture was treated with tra-IR700 and observed (before and after irradiation of NIR-light). Targeted specific necrotic cell death was observed upon excitation with NIR-light $\left(2 \mathrm{~J} / \mathrm{cm}^{2}\right) 30 \mathrm{~min}$ after exposure; 3T3/HER2-luc-GFP cells were stained with dead staining Cytox Blue. No damage was demonstrated in the 3T3-RFP cells. *3T3-RFP cell, $\mathrm{Bar}=25 \mu \mathrm{m}$. J. An almost confluent mix of 3T3/HER2-luc-GFP and 3T3-RFP cells was incubated with tra-IR700 for $6 \mathrm{hr}$ and observed before and after irradiation with NIR-light $\left(2 \mathrm{~J} / \mathrm{cm}^{2}\right)$. Bar $=200 \mu \mathrm{m}$. 
A

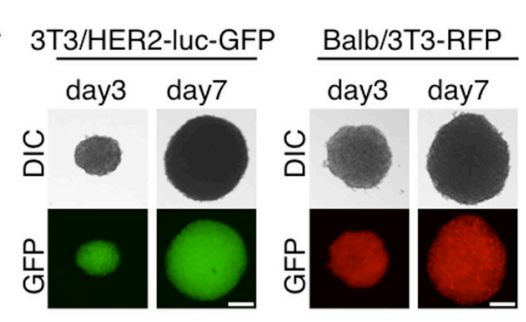

B

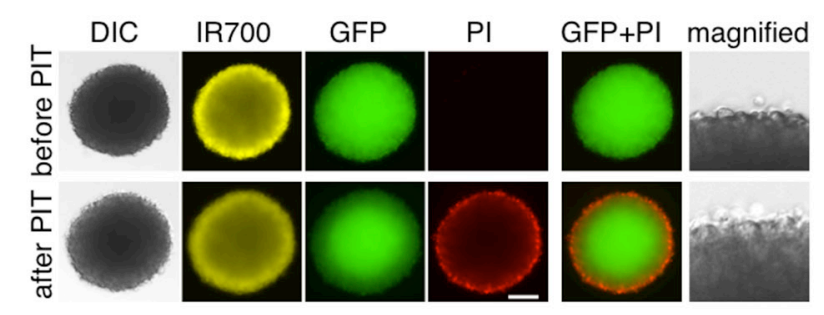

C DIC GFP RFP GFP+RFP
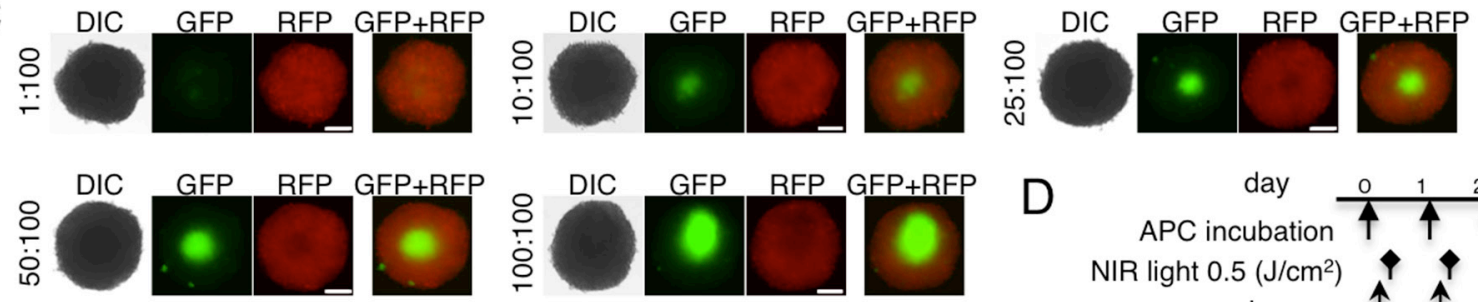

E
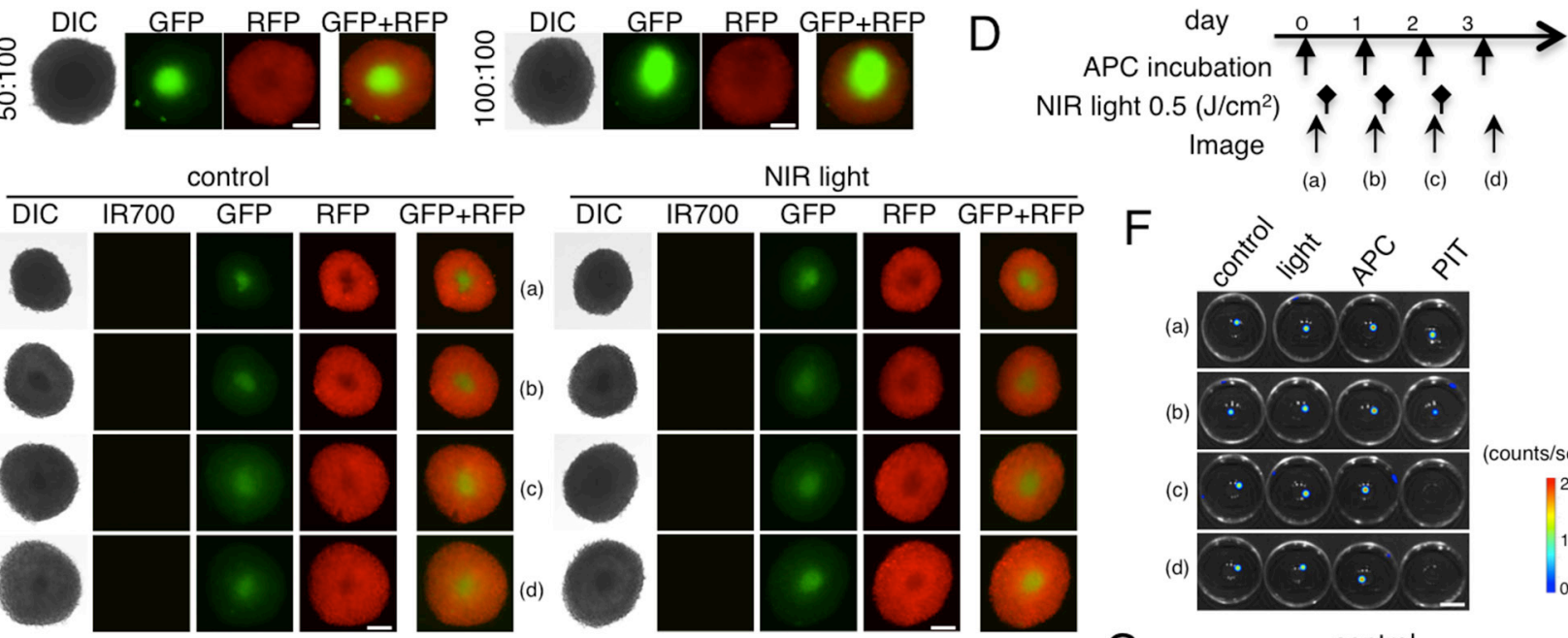

(a)

(b)

(c)

(d)

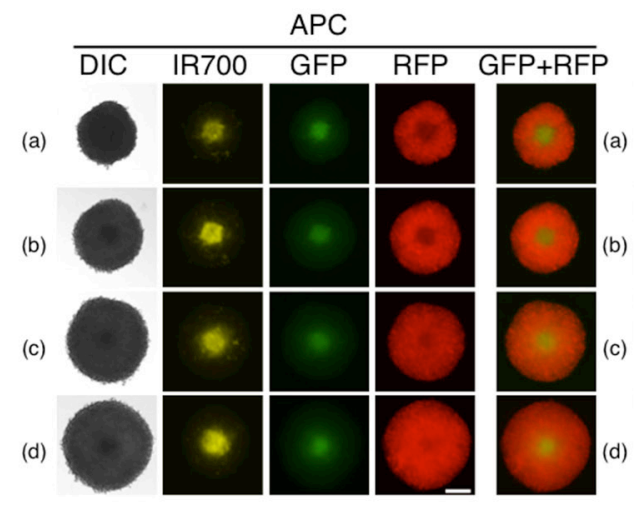

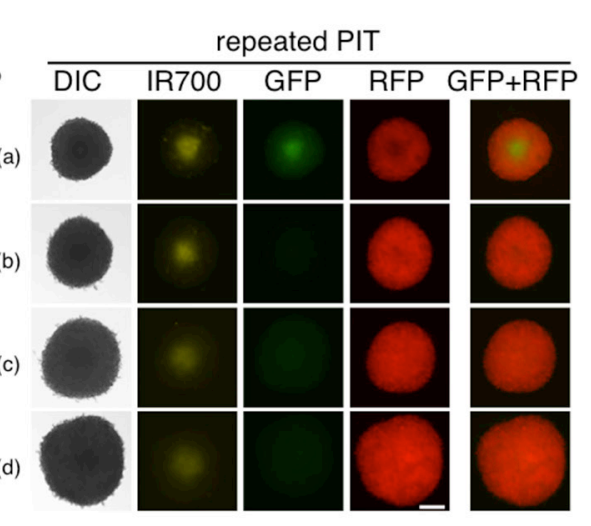

F

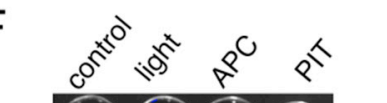

(a)

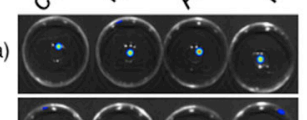

(b)
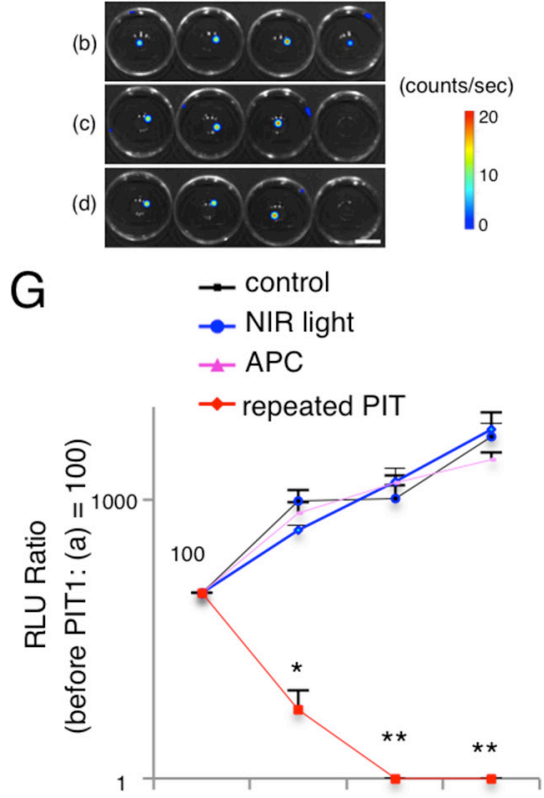

$\begin{array}{llll}\text { (a) } & \text { (b) } & \text { (c) } & \text { (d) }\end{array}$

Figure 2: Evaluation of NIR-PIT effect on 3D cell monoculture and 3D mixed cell culture. A. Representative image of 3T3/ HER2-luc-GFP and 3T3-RFP 3D spheroids at day 3 and day 7. Bar $=200 \mu \mathrm{m}$. B. 3D spheroid at day 7 after $6 \mathrm{hr}$ incubation with tra-IR700, before and after irradiation of NIR-light $\left(0.5 \mathrm{~J} / \mathrm{cm}^{2}\right)$. Necrotic cell death was observed in the layer exhibiting IR700 fluorescence $1 \mathrm{hr}$ after NIR-light. Bar $=200 \mu \mathrm{m}$. C. Representative images of various ratios of mixed spheroid at day 7. Bar $=200 \mu \mathrm{m}$. D. The NIR-PIT regimen incorporating repeated NIR-light exposures is shown. E. Day 7 3T3/HER2-luc-GFP and 3T3-RFP mixed 3D spheroids were divided into 4 groups as shown. GFP fluorescence was greatly diminished after the 2nd NIR-PIT, whereas other cell types grew. Bar $=200 \mu \mathrm{m}$. F. BLI of each group demonstrated that luciferase activity decreased after repeated NIR-PIT. Bar $=10 \mathrm{~mm}$. G. Luciferase activity in mixed 3D spheroids gradually decreased after repeated NIR-PIT, leading to complete killing of cells in the spheroid $(n=5)\left({ }^{*} p=0.0092<0.01\right.$, $* * p<0.0001$ vs control, Tukey's test with ANOVA).

The treatment and imaging regimen is shown (Figure 3A). NIR-irradiation was administered only to the left lung such that the relative effects could be observed in each mouse. The NIR-irradiation effect was confirmed with GFP/RFP-fluorescence or bioluminescence, and there was no obvious difference between the left NIR-irradiation lung and the right lung in each model (Figure 3B, 3C, 3D).

Next, to investigate whether NIR-PIT could specifically prevent metastases, NIR-PIT was administered to the left lung of the mouse models of lung metastases and 
A

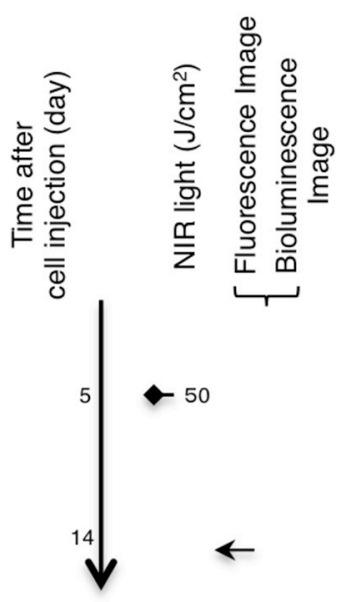

C

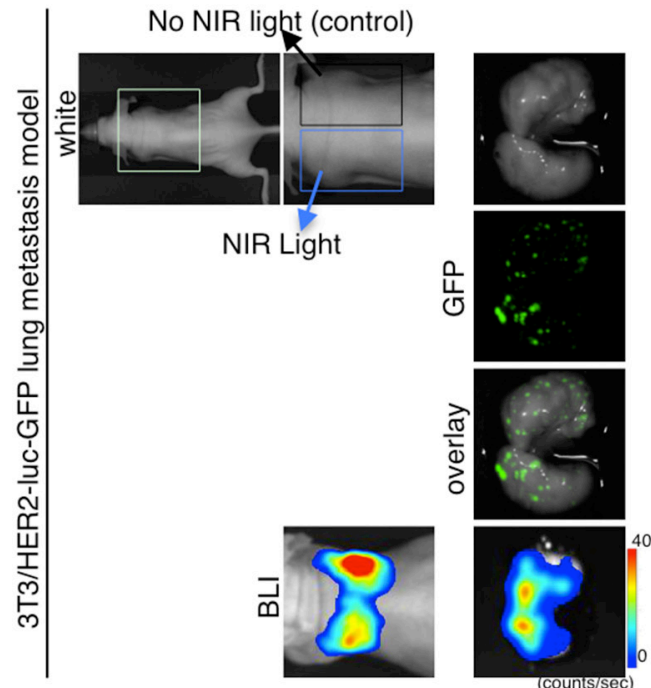

$\mathrm{B}$
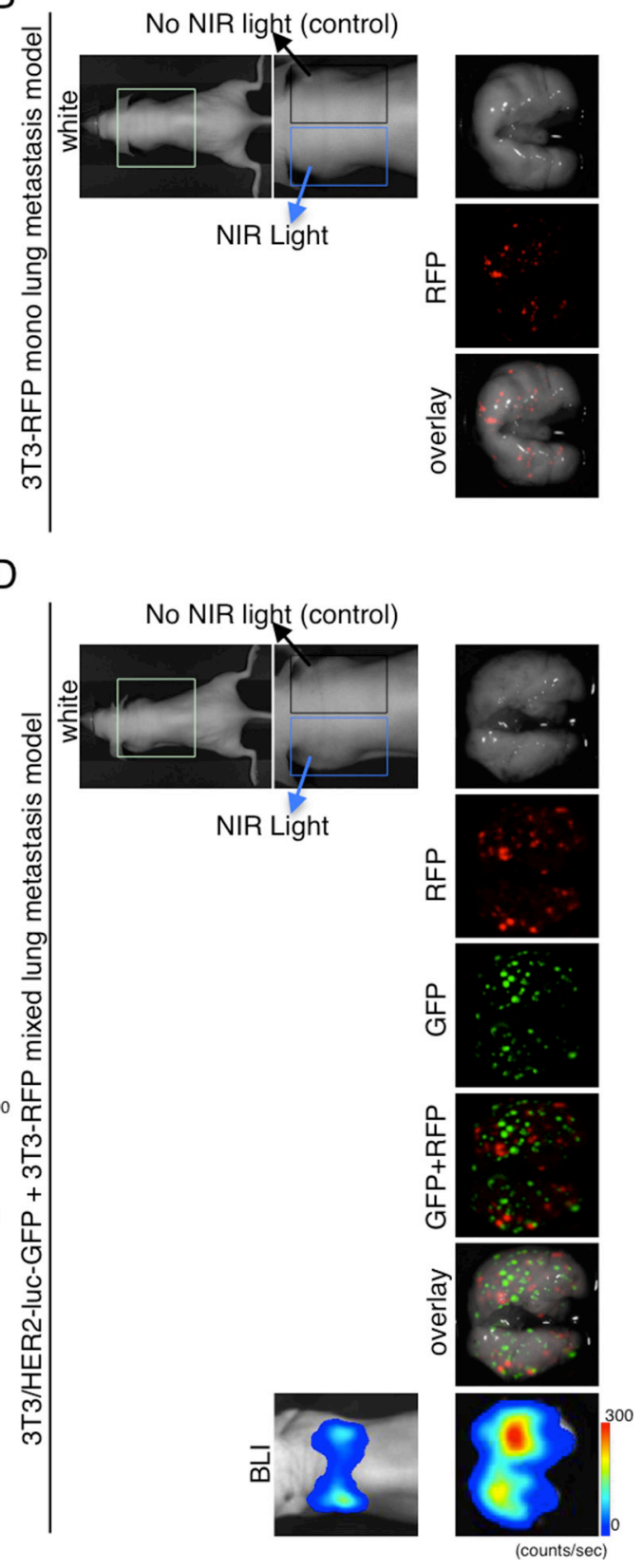

Figure 3: Demonstration of NIR-irradiation effects without intravenous APC injection in lung metastasis model. A. The regimen of NIR-irradiation without intravenous APC injection is shown. Images were obtained at each time point as indicated. NIR-irradiation was administered to the left lung. B. Ex vivo fluorescence imaging (RFP) of 3T3-RFP lung metastasis model. C. In vivo bioluminescence and ex vivo fluorescence/bioluminescence imaging of 3T3/HER2-luc-GFP lung metastasis model. D. In vivo bioluminescence and ex vivo fluorescence/bioluminescence imaging of 3T3/HER2-luc-GFP (target) and 3T3-RFP (non-target) mixed lung metastasis model. B-D. No remarkable preventive effect was detected between the left and right lung. The appearance of both left and right lung was intact.

consisted of 3T3-RFP, 3T3/HER2-luc-GFP, and mixtures of 3T3-RFP and 3T3/HER2-luc-GFP (Figure 4A). NIRPIT was administered only to the left lung such that the relative effects could be observed in each mouse. NIRPIT diminished the IR700-fluorescence on the left side of the chest (Supplemental Figure 1). The NIR-PIT caused decreases in both bioluminescence and GFP-fluorescence in the left lung of the 3T3/HER2-luc-GFP and 3T3-RFP mixed mouse model, but no effect on RFP-fluorescence was detected in the 3T3-RFP and 3T3-RFP+3T3/HER2-luc-GFP mixed lung metastasis mouse model (Figure 4B, 4C, 4D). Moreover, the appearance of the lung gave no indication that NIR-PIT caused damage to the treated left lung. Collectively, these data suggest that NIR-PIT can prevent target-specific metastasis without inducing harm to the normal lung tissue or affecting non-target metastasis formation. 
A

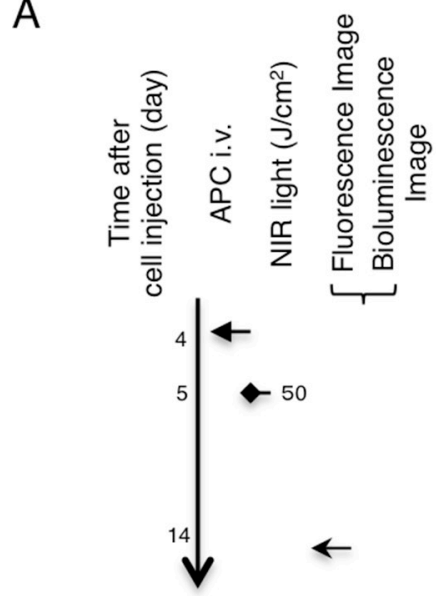

C
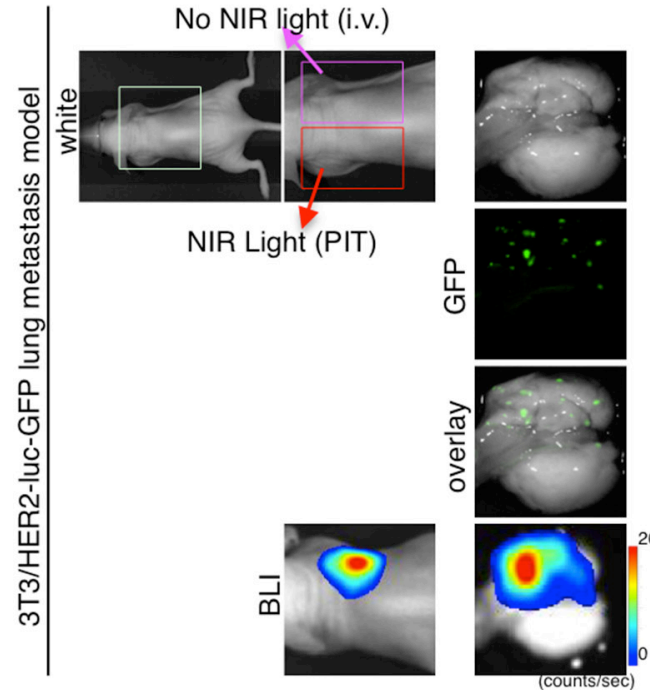

$\mathrm{B}$

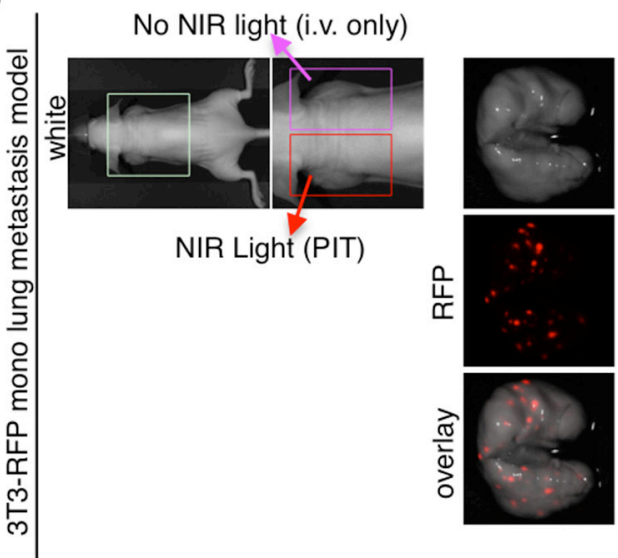

$\mathrm{D}$

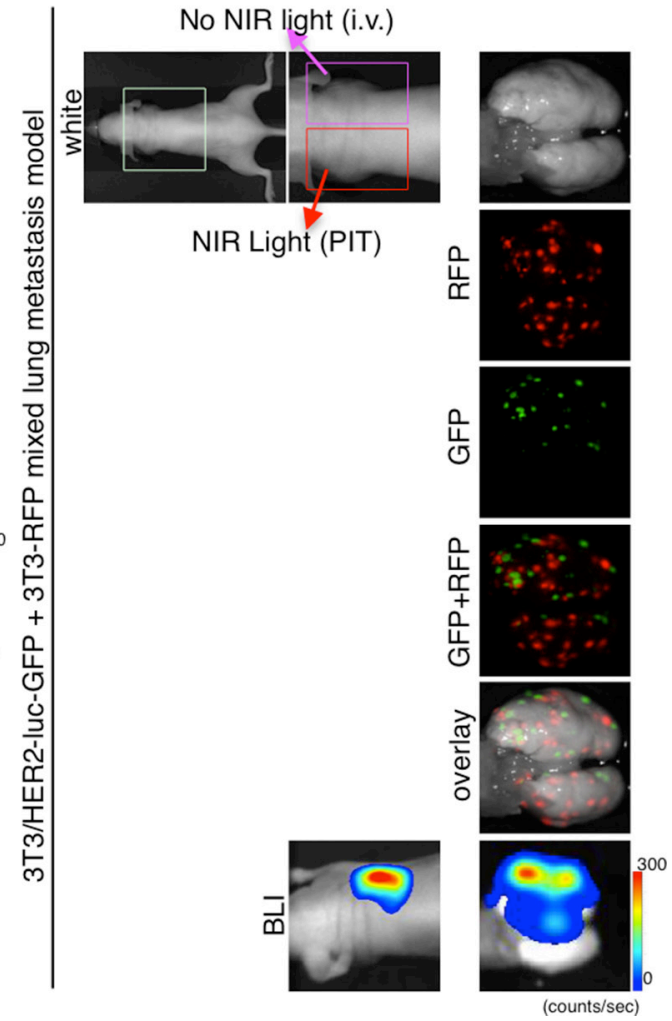

Figure 4: Demonstration of treatment effects of NIR-PIT in lung metastasis model receiving both APC and NIRirradiation. A. The regimen of NIR-PIT is shown. Images were obtained at each time point as indicated. NIR-PIT was administered to the left lung in the same manner as Figure 3. B. Ex vivo fluorescence imaging (RFP) of 3T3-RFP lung metastasis model shows no preventive effect. C. In vivo bioluminescence and ex vivo fluorescence/bioluminescence imaging of 3T3/HER2-luc-GFP lung metastasis model shows distinct decreases of GFP-fluorescence and bioluminescence in the left lung. D. In vivo bioluminescence and ex vivo fluorescence/ bioluminescence imaging of 3T3/HER2-luc-GFP (target) and 3T3-RFP (non-target) mixed lung metastasis model. Remarkable decreases in GFP-fluorescence and bioluminescence in the left lung were observed, but there was no change in RFP-fluorescence between the left and right lung. B-D. The appearance of both left and right lung was intact.

\section{Quantification of the effect of NIR-PIT on lung metastasis model}

To quantify the metastasis prevention effect with NIR-PIT, the luciferase activity, number of metastatic tumor nodules, and GFP/RFP fluorescence between the right and left lung were evaluated in NIR-irradiated and NIR-PIT mice (Figures 3, 4). Luciferase activity was reduced after NIR-PIT in mice with 3T3/HER2-luc-GFP and in the 3T3-RFP+3T3/HER2-luc-GFP mixed lung metastasis mouse model $\left({ }^{*} p<0.0001\right.$, Mann-Whitney $U$-test) (Figure 5A). Numeric evaluation revealed that 
NIR-PIT significantly reduced target GFP tumors, but no significant change was observed in the RFP tumors $\left({ }^{*} p<0.0001\right.$, **n.s., Mann-Whitney $U$-test) (Figure 5B). Finally, fluorescence pixel ratios also showed that NIR-PIT significantly reduced the target GFP-tumor fluorescence pixels compared with non-target RFP-tumor fluorescence pixels $(* p<0.0001, * *$ n.s., Mann-Whitney $U$-test) (Figure 5C). Collectively, these results confirmed that NIR-PIT significantly and selectively reduced lung metastases in this mouse model without damaging the adjacent normal lung tissue or non-target tumors (Figures 3, 4).

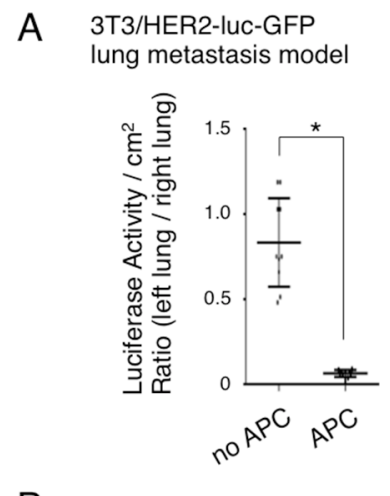

B 3T3-RFP lung metastasis model
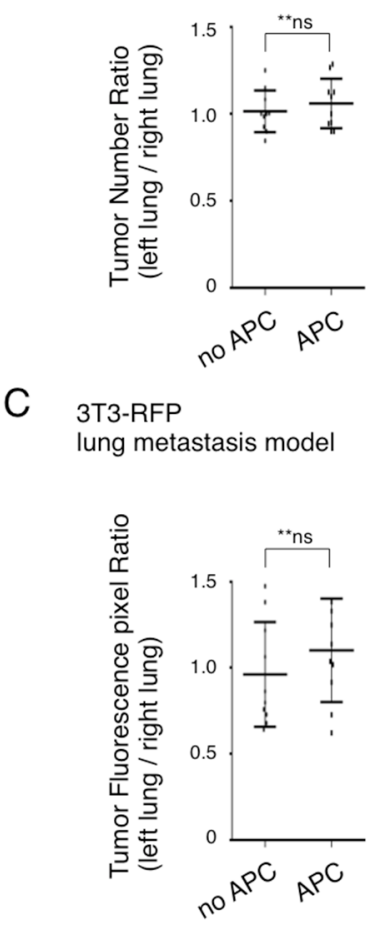

3T3/HER2-luc-GFP + 3T3-RFP mixed lung metastasis model

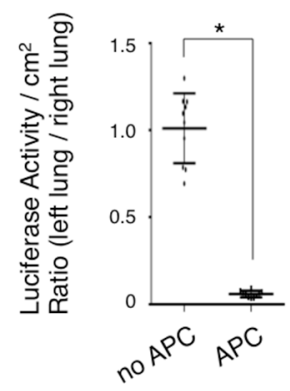

3T3/HER2-luc-GFP lung metastasis model

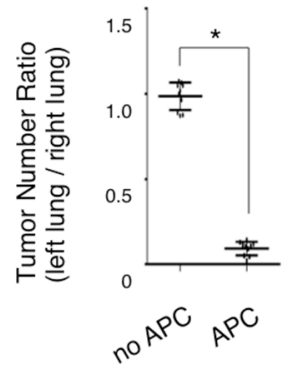

3T3/HER2-luc-GFP lung metastasis model

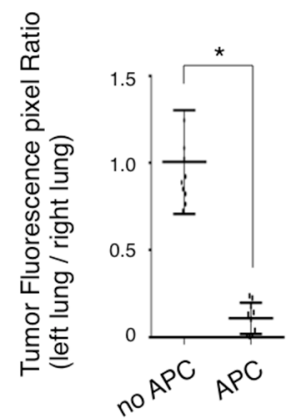

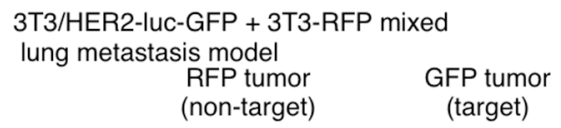
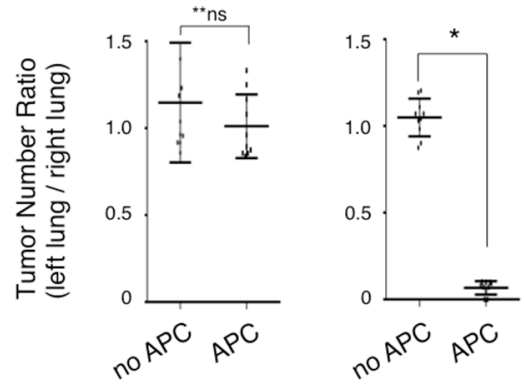

3T3/HER2-luc-GFP + 3T3-RFP mixed lung metastasis model RFP tumo (non-target)

GFP tumor

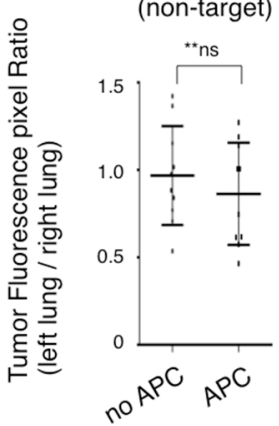

Figure 5: Quantification of NIR-PIT effects on lung metastasis model. A. Quantification of luciferase activity on ex vivo 3T3/ HER2-luc-GFP and 3T3/HER2-luc-GFP and 3T3-RFP mixed metastasis model. Luciferase activity/ $\mathrm{cm}^{2}$ between left and right lung was significantly decreased in the left lung with NIR-PIT ( $\mathrm{n}=10$ in each group) ( ${ }^{*} p<0.0001$, Mann-Whitney $U$-test). B. Quantification of the number of metastases with fluorescence imaging on ex vivo specimens of the 3T3-RFP, 3T3/HER2-luc-GFP, and 3T3/HER2-luc-GFP +3T3-RFP mixed metastasis models. The number of GFP positive tumors was significantly decreased in the left lung after NIR-PIT, whereas the number of RFP positive tumors was unchanged ( $n=10$ in each group) $\left({ }^{*} p<0.0001,{ }^{* *}\right.$ n.s., Mann-Whitney $U$-test). C. Quantification of fluorescence pixels on ex vivo 3T3-RFP, 3T3/HER2-luc-GFP, and 3T3/HER2-luc-GFP and 3T3-RFP mixed metastasis model. GFP fluorescence was significantly decreased in the left lung after NIR-PIT, whereas RFP fluorescence pixels were unchanged $(n=10$ in each group) $\left({ }^{*} p<0.0001, * *\right.$ n.s., Mann-Whitney $U$-test $)$. 


\section{DISCUSSION}

In this study, NIR-PIT significantly and selectively prevented the growth of early lung metastasis in a mouse model. To non-invasively evaluate therapeutic effects in vivo, we used fluorescence and bioluminescence reporters because each modality provided complimentary information. Multimodal imaging has been shown to demonstrate a more nuanced understanding of the therapeutic effects in mice [8-10]. BLI, using firefly luciferase, was used as the primary quantitative outcome measure in this study. This is a well-established method of determining in vivo viability $[11,12]$ because the BLI reaction requires both oxygen and ATP to actively transport the substrate luciferin and subsequently catalyze the photochemical reaction [12]. Because NIR-PIT induced necrotic cell death, which releases cellular ATP, BLI is a good biomarker for the functional effects of NIR-PIT [6,13]. Fluorescence imaging enables the full process of tumorigenesis, treatment, regression, metastasis, or recurrence $[14,15]$. The high level of tumor fluorescence allowed us to perform imaging and quantification of tumor growth and dissemination without using additional injections of contrast agents or substrates. Interestingly, although visible inspection of the lung often indicated no abnormality, fluorescence imaging revealed many invisible early stage metastases [15]. This approach enabled the more precise quantification of the number and fluorescence of tumors in the ex vivo lung. By employing cytoplasmic GFP expressing cells, the antitumor effects induced by NIR-PIT could be clearly monitored due to the extrusion of GFP from treated cells [7]. RFP fluorescence was not significantly affected because it was expressed only in non-target cells, which enabled the confirmation of highly selective cell killing. Thus, with this combination of in vivo imaging, we demonstrated the significant and selective prevention of lung metastases.

We used a lung metastasis model that utilized tail vein cell injection [16]. Surgical orthotopic injection (SOI) is another method of creating a lung metastasis model [17]. Various advantages and disadvantages exist between these models. SOI requires highly trained skills and invasive methods and results in high pre-procedure mortality rate, but it is thought to be a more physiological model. The cell-injection model has the advantages of less advanced skills, less invasiveness to animals, and a very low preprocedure mortality rate. Due to these considerations and the recent requirement of animal wellness, we used cellinjection models. Moreover, in a mice lung metastasis model, it is difficult to observe live fluorescence imaging because the mice die due to pneumothorax. To prevent this pneumothorax, a ventilation system for mice was necessary, and it demanded advanced skills, invasive procedures, and expensive devices [18].Thus, we monitored live cell-injection lung metastasis models by non-invasive BLI and then confirmed our findings with ex vivo fluorescence imaging.
The metastatic process consists of several steps: 1. loss of cellular adhesion, increased motility and invasiveness, which leads to 2 . intravasation into the circulatory system, 3. transport by circulation, 4 . adherence to distant organ vessel walls, 5. extravasation, and 6. colonization at a distant site [19-22]. Despite recent advances in the understanding of this process, metastases remain the major cause of cancer-associated mortality. Unfortunately, systemic therapies that are administered pre-emptively are typically unsuccessful in suppressing metastases [19]. In this study, by targeting metastatic cancer cells at steps 4, 5, and 6 described above, NIR-PIT was able to prevent the establishment and/or growth of metastases in the lung. NIR-PIT can treat cancer cells that have access to the APC and NIR-light. Thus, in the vessel, where tumor cells are at the time of extravasation and at colonization, they are susceptible to NIR-PIT, particularly in the lung, where the transmission of light is optimal.

In addition to metastases, NIR-PIT could be used at the primary site, especially during or after surgery to "mop up" residual cancer cells. This therapy could also treat circulating tumor cells (CTCs), at least within the superficial vessels such as arteries and veins in the skin. The ability to prevent the growth of metastases is another possible role for NIR-PIT.

Some malignancies, such as sarcomas, preferentially metastasize to the lungs [23]. This factor has prompted the use of surgical removal of oligometastases as a strategy to prolong survival. It is possible that NIR-PIT could be particularly useful in these tumor types as a method for preventing pulmonary metastases. Naturally, this approach would require the identification of an antibody reliably associated with sarcomas [24]. Unlike the mouse, in which NIR-light can be administered transcutaneously, in the human, NIR-light would likely be administered bronchoscopically or thoracoscopically. Compared with surgery, NIR-PIT has the distinct advantage of minimal invasiveness and not incurring damage to the normal lung parenchyma. It could also be repeated multiple times in the same patient if suspicions of additional pulmonary metastases arise. This process could enable the preservation of pulmonary reserve in addition to reducing, if not eliminating, the impact of metastatic disease. Of course, NIR-PIT would have less impact if there were other sites of metastases that were less amenable to NIR-PIT.

There are several limitations to this study. First, few pulmonary metastases overexpress HER2, which was simply used as an exemplar in this study. However, NIR-PIT has proven to be effective with multiple antibodies, including anti-EGFR, anti-PSMA, antimesothelin, and anti-CEA [5-7, 13, 25, 26]. Therefore, we are optimistic that NIR-PIT could be used with a wide range of antibodies and cancers. The possibility of mixtures of APCs is also feasible in cases in which there are mixed populations of antigen expressing cells [27]. 
Due to limited light penetration, translation to humans would require bronchoscopy or direct thoracoscopy with fibrooptics to expose light to the entire lung. Metastases outside the lungs would not be affected by NIR-PIT, and therefore, the procedure may have little impact in widespread metastatic disease. However, because the lungs are often the most symptomatic, NIR-PIT may provide palliative relief and modest survival benefits in the presence of extrapulmonary metastases. Another limitation is that the long-term durability of response and side effects of NIR-PIT were not determined. However, short-term studies of the mice demonstrated only temporary minimal body weight loss after NIR-PIT.

In conclusion, this study demonstrates that NIRPIT can be used as a local treatment of early pulmonary metastases and can therefore be viewed as a preventive therapy for pulmonary metastases.

\section{MATERIALS AND METHODS}

\section{Reagents}

Water soluble, silicon-phthalocyanine derivative, IRDye 700DX NHS ester was obtained from LI-COR Bioscience (Lincoln, NE, USA). Trastuzumab, $95 \%$ humanized $\mathrm{IgG}_{1} \quad \mathrm{mAb}$ directed against HER2, was purchased from Genentech (South San Francisco, CA, USA). All other chemicals were of reagent grade.

\section{Synthesis of IR700-conjugated trastuzumab}

Conjugation of dyes with mAbs was performed according to previous reports [13]. Briefly, trastuzumab (1 mg, $6.8 \mathrm{nmol}$ ) was incubated with IR700 NHS ester $(60.2 \mu \mathrm{g}, 30.8 \mathrm{nmol})$ in $0.1 \mathrm{~mol} / \mathrm{L} \mathrm{Na}_{2} \mathrm{HPO}_{4}(\mathrm{pH} \mathrm{8.6})$ at room temperature for $1 \mathrm{hr}$. The mixture was purified with a Sephadex G25 column (PD-10; GE Healthcare, Piscataway, NJ, USA). The protein concentration was determined with the Coomassie Plus protein assay kit (Thermo Fisher Scientific Inc, Rockford, IL, USA) by measuring absorption at $595 \mathrm{~nm}$ (8453 Value System; Agilent Technologies, Santa Clara, CA, USA). The concentration of IR700 was measured by absorption at $689 \mathrm{~nm}$ to confirm the number of fluorophore molecules conjugated to each $\mathrm{mAb}$. The synthesis was controlled such that an average of three IR700 molecules were bound to a single antibody. We performed SDS-PAGE as a quality control for each conjugate as previously reported [5]. We abbreviate IR700 conjugated to trastuzumab as tra-IR700.

\section{Cell culture}

HER2 and luciferase/GFP-expressing NIH3T3 cells (3T3/HER2-luc-GFP) were established with a transfection of RediFect Red-FLuc-GFP (PerkinElmer, Waltham, MA, USA). High GFP and luciferase expression was confirmed with 10 passages. Balb/3T3 cells stably expressing RFP were established with transfection by RFP (EF1a)-Neo lentiviral particles (AMSBIO, Cambridge, MA, USA). High RFP expression was confirmed in the absence of a selection agent with 10 passages. 3T3 cells stably expressing RFP (3T3-RFP) were used as negative controls [13]. Cells were grown in RPMI 1640 (Life Technologies, Gaithersburg, MD, USA) supplemented with 10\% fetal bovine serum and $1 \%$ penicillin/streptomycin (Life Technologies) in tissue culture flasks in a humidified incubator at $37^{\circ} \mathrm{C}$ at an atmosphere of $95 \%$ air and $5 \%$ carbon dioxide.

\section{D spheroid culture}

Spheroids were generated by the hanging drop method in which five thousand cells were suspended in $50 \mu \mathrm{L}$ medium and were then dispensed into 96 well plates (3D Biomatrix Inc, Ann Arbor, MI, USA) following the manufacturer's instructions [13]. Mixed spheroids were made with 5,000 cells of Balb/3T3-RFP and 500 cells of 3T3/HER2-luc-GFP (100:10). After observation or treatment, spheroids were again incubated with the hanging drop plates containing new media.

\section{Flow cytometry}

Fluorescence from cells incubated with tra-IR700, GFP fluorescence or RFP fluorescence was measured using a flow cytometer (FACS Calibur, BD BioSciences, San Jose, CA, USA) and analyzed with CellQuest software (BD BioSciences). 3T3/HER2-luc-GFP or 3T3RFP cells $\left(1 \times 10^{5}\right)$ were incubated with tra-IR700 for $6 \mathrm{hr}$ at $37^{\circ} \mathrm{C}$. To validate the specific binding of the conjugated antibody, excess antibody $(50 \mu \mathrm{g})$ was used to block $0.5 \mu \mathrm{g}$ of APCs [6].

\section{Fluorescence microscopy}

To detect the antigen specific localization of IR700 conjugates, fluorescence microscopy was performed (IX81; Olympus America, Melville, NY, USA). Ten thousand cells were seeded on cover-glass-bottomed dishes and incubated for $24 \mathrm{hr}$. Tra-IR700 was then added to the culture medium at $10 \mu \mathrm{g} / \mathrm{mL}$ and incubated at $37^{\circ} \mathrm{C}$ for $6 \mathrm{hr}$. The cells were then washed with PBS; Propidium Iodide (PI) (1:2000) (Life Technologies) and Cytox Blue (1:500) (Life Technologies) were used to detect dead cells. They were added into the media $30 \mathrm{~min}$ before observation. The cells were then exposed to NIR-light $\left(2 \mathrm{~J} / \mathrm{cm}^{2}\right)$, and serial images were obtained. The filter was set to detect IR700 fluorescence using a 590-650 $\mathrm{nm}$ excitation filter and a $665-740 \mathrm{~nm}$ band pass emission filter. 
The images were analyzed using the Image J software (http://rsb.info.nih.gov/ij/) [28].

\section{In vitro NIR-PIT}

One hundred thousand cells were seeded into 24-well plates, or ten million cells were seeded into a $10 \mathrm{~cm}$ dish and incubated for $24 \mathrm{hr}$. The medium was replaced with fresh culture medium containing $10 \mu \mathrm{g} / \mathrm{mL}$ of tra-IR700 that was incubated for $6 \mathrm{hr}$ at $37^{\circ} \mathrm{C}$. After washing with PBS, phenol red free culture medium was added. Then, cells were irradiated with a NIR laser, which emits light at a 685 to $695 \mathrm{~nm}$ wavelength (BWF5-690-8-600-0.37; B\&W TEK INC., Newark, DE, USA). The output power density in $\mathrm{mW} / \mathrm{cm}^{2}$ was measured with an optical power meter (PM 100, Thorlabs, Newton, NJ, USA).

\section{Cytotoxicity/Phototoxicity assay}

The cytotoxic effects of NIR-PIT with tra-IR700 were determined by luciferase activity and flow cytometry of PI staining. For luciferase activity, $150 \mu \mathrm{g} / \mathrm{mL}$ of D-luciferin-containing media (Gold Biotechnology, St Louis, MO, USA) was administered to PBS-washed cells $1 \mathrm{hr}$ after NIR-PIT, which were analyzed on a bioluminescence imaging (BLI) system (Photon Imager; Biospace Lab, Paris, France). For the flow cytometry assay, cells were trypsinized $1 \mathrm{hr}$ after treatment and washed with PBS. PI was added to the cell suspension (final $2 \mu \mathrm{g} / \mathrm{mL}$ ), and the cells were incubated at room temperature for 30 min prior to flow cytometry. Residual GFP fluorescence at $1 \mathrm{hr}$ after NIR-PIT was also evaluated with FACS [7].

\section{Animal and tumor models}

All in vivo procedures were conducted in compliance with the Guide for the Care and Use of Laboratory Animal Resources (1996), US National Research Council, and approved by the local Animal Care and Use Committee. Six- to eight-week-old female homozygote athymic nude mice were purchased from Charles River (NCI-Frederick). During the procedures, mice were anesthetized with isoflurane.

To evaluate targeted metastasis prevention with NIR-PIT in the lung metastasis mouse model [16], two million 3T3/HER2-luc-GFP cells in PBS (total $200 \mu \mathrm{L}$ ) for mono-lung metastasis were intravenously injected through the tail vein. Two million 3T3-RFP cells in PBS (total $200 \mu \mathrm{L}$ ) for the 3T3-RFP lung metastasis mouse model and a mixture of one million 3T3/HER2-luc-GFP cells and one million 3T3-RFP cells in PBS (total $200 \mu \mathrm{L}$ ) for the mixed lung metastasis mouse model were intravenously injected through the tail vein.

\section{In vivo fluorescence imaging}

In vivo fluorescence images were obtained using a Pearl Imager (LI-COR Bioscience) to detect IR700 fluorescence, and a Maestro Imager (CRi, Woburn, MA, USA) was used to detect GFP/RFP. A band-pass filter from 445 to $490 \mathrm{~nm}$ (excitation), and a long-pass blue filter over $515 \mathrm{~nm}$ (emission) was used for GFP analysis; 503 to $555 \mathrm{~nm}$ (excitation) and a long-pass green filter over $580 \mathrm{~nm}$ (emission) were used for RFP analysis. The tunable emission filter was automatically increased in $10-\mathrm{nm}$ increments from 515 to $580 \mathrm{~nm}$ at a constant exposure time $(800 \mathrm{msec})$. The spectral fluorescence images consisted of autofluorescence spectra and the spectra from GFP/RFP (tumor), which were then unmixed based on the characteristic spectral pattern of GFP using Maestro software (CRi). Regions of interest (ROIs) were manually drawn on either the left or right lung, as appropriate to the model, and the fluorescence intensity and pixels were measured [7]. The number of the tumor nodules in the left or right lung was calculated.

\section{In vivo bioluminescence imaging}

For BLI, D-luciferin $(15 \mathrm{mg} / \mathrm{mL}, 200 \mu \mathrm{L})$ was injected intraperitoneally, and the mice were analyzed with a Photon Imager for luciferase activity at day 14 . To quantify the luciferase activities, ROIs of the entire left lung and entire right lung in vivo/ ex vivo were obtained. To account for the differences in volume between the left and right lung, the signal was recorded as photon counts $/ \mathrm{cm}^{2}$.

\section{In vivo NIR-PIT}

Mice were randomized into 2 groups of 10 animals and divided into four groups based on local NIR-irradiation (in the left or right lung) for the following treatments (see Figure 3A, 4A): (1) right lung with no treatment (control); (2) left lung with local NIR-light exposure only at $50 \mathrm{~J} / \mathrm{cm}^{2}$ on day 5 after cell injection; (3) right lung with $100 \mu \mathrm{g}$ of tra-IR700 i.v. on day 4 but no NIR-light exposure on day 5 after cell injection; and (4) left lung with $100 \mu \mathrm{g}$ of tra-IR700 i.v. on day 4 after cell injection followed by NIR-light at $50 \mathrm{~J} / \mathrm{cm}^{2}$ one day after intravenous injection of tra-IR700 (treatment group). The mice were irradiated with NIR-light from 2 directions (each $25 \mathrm{~J} / \mathrm{cm}^{2}$ ) via the left back and left chest. IR700 fluorescence imaging was performed before and after NIR-PIT. Serial fluorescence imaging (RFP/GFP) and BLI were obtained at 14 days after cell injection (see Figures 3A, 4A).

\section{Statistical analysis}

Data are expressed as the means \pm s.e.m. from a minimum of four experiments, unless otherwise indicated. 
Statistical analyses were performed using a statistics program (GraphPad Prism; GraphPad Software, La Jolla, CA, USA). For multiple comparisons, a one-way analysis of variance (ANOVA) with Tukey's test was used. Student's $t$ test was used to compare the two in vitro $2 \mathrm{D}$ culture studies. To compare the tumor left/right ratio in the lung, a Mann-Whitney $U$-test was used. $p<0.05$ was considered to indicate a statistically significant difference.

\section{DISCLOSURE STATEMENT}

None declared.

\section{GRANT SUPPORT}

This research was supported by the Intramural Research Program of the National Institutes of Health, National Cancer Institute, Center for Cancer Research. K.S. is supported with JSPS Research Fellowship for Japanese Biomedical and Behavioral Researchers at NIH.

\section{Author contributions}

K.S. mainly conducted the experiments, created the study concept, performed the analysis and wrote the manuscript; P.L.C. wrote the manuscript and supervised the project; and H.K. planned and initiated the project, designed and conducted experiments, wrote the manuscript, and supervised the entire project.

\section{REFERENCES}

1. Weichselbaum RR, Hellman S. Oligometastases revisited. Nat Rev Clin Oncol. 2011; 8:378-82. doi:10.1038/ nrclinonc.2011.44.

2. Steeg PS. Tumor metastasis: mechanistic insights and clinical challenges. Nat Med. 2006; 12:895-904. doi:10.1038/nm1469.

3. Mew D, Wat C, Towers GHN, Levy JG. Photoimmunotherapy: treatment of animal tumors with tumor-specific monoclonal antibody-hematoporphyrin conjugates. J Immunol. 1983; 130:1473-7.

4. Oseroff AR, Ohuoha D, Hasan T, Bommer JC, Yarmush ML. Antibody-targeted photolysis: selective photodestruction of human T-cell leukemia cells using monoclonal antibody-chlorin e6 conjugates. Proc Natl Acad Sci U S A. 1986; 83:8744-8.

5. Mitsunaga M, Ogawa M, Kosaka N, Rosenblum LT, Choyke PL, Kobayashi H. Cancer cell - selective in vivo near infrared photoimmunotherapy targeting specific membrane molecules. Nat Med. 2011; 17:1685-91. doi: $10.1038 / \mathrm{nm} .2554$.

6. Sato K, Watanabe R, Hanaoka H, Harada T, Nakajima T, Kim I, Paik CH, Choyke PL, Kobayashi H.
Photoimmunotherapy: Comparative effectiveness of two monoclonal antibodies targeting the epidermal growth factor receptor. Mol Oncol. 2014; 8:620-32. doi:10.1016/j. molonc.2014.01.006.

7. Sato K, Choyke PL, Kobayashi H. Photoimmunotherapy of Gastric Cancer Peritoneal Carcinomatosis in a Mouse Model. PLoS One. 2014; 9:e113276. doi:10.1371/journal. pone. 0113276.

8. Francia G, Cruz-Munoz W, Man S, Xu P, Kerbel RS. Mouse models of advanced spontaneous metastasis for experimental therapeutics. Nat Rev Cancer. 2011; 11:135-41. doi:10.1038/nrc3001.

9. Conway JRW, Carragher NO, Timpson P. Developments in preclinical cancer imaging: innovating the discovery of therapeutics. Nat Rev Cancer. 2014; 14:314-28. doi: $10.1038 / \operatorname{nrc} 3724$.

10. De Jong $M$, Essers $J$, van Weerden WM. Imaging preclinical tumour models: improving translational power. Nat Rev Cancer. 2014; 14:481-93. doi:10.1038/nrc3751.

11. Contag PR, Olomu IN, Stevenson DK CC. Bioluminescent indicators in living mammals. Nat Med. 1998; 4:245-7.

12. Rehemtulla A, Stegman LD, Cardozo SJ, Gupta S, Hall DE, Contag CH, Ross BD. Rapid and Quantitative Assessment of Cancer Treatment Response Using In Vivo Bioluminescence Imaging 1 Days post sham treatment. Neoplasia. 2000; 2:491-5.

13. Sato K, Hanaoka H, Watanabe R, Nakajima T, Choyke PL, Kobayashi H. Near infrared photoimmunotherapy in the treatment of disseminated peritoneal ovarian cancer. Mol Cancer Ther. 2015; Jan 14:141-50. doi: 10.1158/15357163.MCT-14-0658. Epub 2014 Nov 21.

14. Hoffman RM. The multiple uses of fluorescent proteins to visualize cancer in vivo. Nat Rev Cancer. 2005; 5:796-806. doi:10.1038/nrc1717.

15. Yamamoto N, Tsuchiya H, Hoffman RM. Tumor imaging with multicolor fluorescent protein expression. Int J Clin Oncol. 2011; 16:84-91. doi:10.1007/s10147-011-0201-y.

16. Koyama Y, Hama Y, Urano Y, Nguyen DM, Choyke PL, Kobayashi H. Spectral fluorescence molecular imaging of lung metastases targeting HER2/neu. Clin cancer Res. 2007; 13:2936-45. doi:10.1158/1078-0432.CCR-06-2240.

17. Yang $M$, Hasegawa S, Jiang $\mathrm{P}$, Wang $\mathrm{X}$, Tan $\mathrm{Y}$, Chishima T, Shimada H, Moossa AR, Hoffman RM. Widespread skeletal metastatic potential of human lung cancer revealed by green fluorescent protein expression. Cancer Res. 1998; 58:4217-21.

18. Kimura H, Hayashi K, Yamauchi K, Yamamoto N, Tsuchiya H, Tomita K, Kishimoto H, Bouvet M, Hoffman RM. Real-time imaging of single cancer-cell dynamics of lung metastasis. J Cell Biochem. 2010; 109:58-64. doi:10.1002/jcb.22379.

19. Chaffer CL, Weinberg RA. A perspective on cancer cell metastasis. Science. 2011; 331:1559-64. doi:10.1126/ science. 1203543. 
20. Chambers AF, Groom AC, MacDonald IC. Dissemination and growth of cancer cells in metastatic sites. Nat Rev Cancer. 2002; 2:563-72. doi:10.1038/nrc865.

21. Fidler IJ. hypothesis revisited. Nat Rev Cancer. 2003; 3:1-6.

22. Sethi N, Kang Y. Unravelling the complexity of metastasis - molecular understanding and targeted therapies. Nat Rev Cancer. 2011; 11:735-48. doi:10.1038/nrc3125.

23. Nguyen DX, Bos PD, Massagué J. Metastasis: from dissemination to organ-specific colonization. Nat Rev Cancer. 2009; 9:274-84. doi:10.1038/nrc2622.

24. Treasure $T$, Milošević M, Fiorentino F, Macbeth F. Pulmonary metastasectomy: what is the practice and where is the evidence for effectiveness?. Thorax. 2014; 69:946-9. doi:10.1136/thoraxjnl-2013-204528.

25. Shirasu N, Yamada H, Shibaguchi H, Kuroki M, Kuroki M. Potent and specific antitumor effect of CEA-targeted photoimmunotherapy. Int J Cancer. 2014; 135:2697-710. doi:10.1002/ijc.28907.

26. Sato K, Nagaya T, Choyke PL, Kobayashi H. Near Infrared Photoimmunotherapy in the Treatment of Pleural Disseminated NSCLC: Preclinical Experience. Theranostics. 2015; 5: 698-709. doi: 10.7150/thno.11559.

27. Nakajima T, Sano K, Choyke PL, Kobayashi H. Improving the efficacy of Photoimmunotherapy (PIT) using a cocktail of antibody conjugates in a multiple antigen tumor model. Theranostics. 2013; 3:357-65. doi:10.7150/thno.5908.

28. Sato K, Watanabe T, Wang S, Kakeno M, Matsuzawa K, Matsui T, Yokoi K, Murase K, Sugiyama I, Ozawa M, Kaibuchi K. Numb controls E-cadherin endocytosis through p120 catenin with aPKC. Mol Biol Cell. 2011; 22:3103-19. doi:10.1091/mbc.E11-03-0274. 